\title{
LAS CREENCIAS DE DOCENTES MEXICANOS SOBRE EL PAPEL DE LA ESCUELA Y DEL
}

\section{MAESTRO $^{1}$}

\author{
SILVIA MACOTELA FLORES \\ ROSA DEL CARMEN FLORES MACÍAS \\ ILEANA SEDA SANTANA \\ Facultad de Psicología \\ Universidad Nacional Autónoma de México
}

\section{Introducción}

El presente trabajo que forma parte de un proyecto más amplio (Macotela, Seda y Flores, 1997), se inserta en una problemática que surge recientemente en el sistema educativo mexicano a raíz de cambios substanciales en la manera de conceptuar la Educación Especial en el nivel internacional. Este movimiento, denominado como "integración educativa", parte de un modelo que privilegia la igualdad de oportunidades educativas para todos los alumnos para lo que establece al aula regular como el espacio idóneo para que se cumplan esos fines (Ainscow, 1995).

Los cambios generados por la integración educativa desde principios de la década de los 90 fueron tanto de carácter político como administrativo. Es importante destacar que hasta que se aprobó la Ley de Integración Educativa, en México los servicios de educación especial y los de educación regular, operaban de manera independiente. Los servicios de educación especial se impartían no sólo fuera de las aulas sino fuera de las escuelas regulares, imperando un modelo más de tipo asistencial que educativo.

La nueva política permite conceptuar a la educación especial como una modalidad de la educación básica (que en México abarca los niveles de educación preescolar, primaria y secundaria), y postula la determinación de las necesidades educativas especiales en este contexto (SEP, 1994) A partir de 1993 se intensifican los esfuerzos para que se reconozca a los alumnos con necesidades educativas especiales como parte del alumnado de educación regular. En consecuencia se replantean los propósitos y mecanismos de los servicios de educación especial con objeto de transformarse en un apoyo directo a la educación regular.

En esta perspectiva, la exigencia para el docente de aula regular consiste en que asuma la responsabilidad de atender las demandas de los niños con necesidades educativas especiales,

\footnotetext{
${ }^{1}$ El presente reporte forma parte del proyecto "Desarrollo y evaluación de un programa modelo de colaboración entre maestros de aula y maestros de apoyo y su relación con el logro académico en niños de primaria”, financiado por CONACYT (Ref. 23369-H)
} 
Macotela Flores, Silvia; Flores Macías, Rosa del Cármen; Seda Santana, lleana: Las Creencias de ...

quienes anteriormente eran atendidos exclusivamente por los servicios de educación especial. Por su parte, se demanda al maestro de educación especial apoyar a la educación regular en colaboración con los maestros de aula. Esto es, dos instancias que anteriormente funcionaban y brindaban servicios de manera independiente, ahora requieren trabajar en mutua colaboración.

Sin embargo, la política educativa no se traduce de inmediato en acciones concretas o cambios notorios, más bien forma parte de un proceso de intentos y ajustes a largo plazo. Esto es particularmente cierto cuando se trata de reconceptuar las prácticas de trabajo de los profesionales de la educación de acuerdo con las nuevas demandas impuestas por los cambios en las políticas educativas.

Gimeno Sacristán (1989), con referencia a la implantación del curriculum, ha reiterado la importancia de tomar en cuenta la mediación de los docentes y el papel que ésta juega en la práctica educativa. El movimiento de integración educativa no es la excepción, para hacerlo realidad, se requiere de la mediación de los docentes, en este caso de los maestros de aula (de educación regular) y los maestros de apoyo (de educación especial) quienes de acuerdo con la nueva política educativa deberán modificar significativamente su práctica educativa. Lo anterior ha traído como consecuencia confusión y resistencia por parte de ambos grupos de maestros, lo que es predecible ante situaciones de cambio.

Es en este contexto que se inserta el presente trabajo ubicando como interés principal explorar el pensamiento de los docentes acerca de lo que para ellos significa la escuela como institución, el papel de la escuela en la sociedad, y su propia función como docentes. Esto incluye dimensiones relacionadas con sus creencias y las repercusiones que las mismas tienen en sus prácticas de enseñanza (Borko \& Putnam, 1996; Calderhead, 1996)

\section{Perspectiva Teórica}

En México, al igual que en otros países se ha reconocido a los docentes como el eje del sistema educativo. Así se estableció en el Acuerdo Nacional Para la Modernización Educativa (SEP-SNTE, 1989), y como resultado se propusieron acciones conducentes a reconocer al magisterio como elemento esencial. Similarmente, se establece explícitamente que el elemento central para la implantación y el éxito de las políticas educativas centradas en mejorar la calidad de la educación, es el docente.

Mejorar la calidad de la educación es una meta que ocupa a los gobiernos de muchas naciones. Como resultado, los maestros y los formadores de maestros han quedado en la mira 
Macotela Flores, Silvia; Flores Macías, Rosa del Cármen; Seda Santana, lleana: Las Creencias de ...

como un medio para reformar la educación. Simultáneamente, las disciplinas interesadas en la educación han ido desarrollando investigaciones acerca de las creencias de los docentes.

Los estudios sobre las creencias de los maestros (en algunos estudios denominadas como "teorías implícitas") han intentado entender de mejor manera la práctica educativa. El término "creencias" surge en la investigación educativa como un constructor para comprender e interpretar las acciones de los maestros (De Witt, Birrell, Egan, Cook, Ostlund \& Young, 1998; Knowles \& Cole, 1994; Holt-Reynolds, 1992). El hilo conductor de todos éstos trabajos ha sido conocer y comprender para propiciar el cambio. Esto es, explicitar lo que tradicionalmente se ha mantenido implícito con una primera intención de desarrollar estrategias conducentes a cambiar creencias y una segunda y final intención de lograr cambios duraderos y de fondo en la educación (Maxson \& Sindelar, 1998).

Algunas de las investigaciones se han centrado en los alumnos de escuelas formadoras de maestros, otras en los formadores mismos como es el caso del estudio de De Witt, y colaboradores (1998). Otros autores se han referido a las creencias de los maestros en servicio (Breck, 1995; Fear, 1995), principalmente con la intención de entenderlas para promover cambios en las mismas.

Knowles (1994) y Pajeras (1992) han reiterado que las creencias acerca de la escuela y de la enseñanza se establecen muy temprano en la vida de los individuos por medio de la experiencia misma de la escolarización. Por lo tanto, son resistentes al cambio aún cuando los maestros hayan estudiado en escuelas formadoras de docentes. Por su parte, Hollingsworth en $1989 \mathrm{y}$ Lortie en 1975 habian señalado que las creencias, por desconocidas, pueden ser uno de los principales factores para perpetuar prácticas educativas arcaicas y poco efectivas. Similarmente reiteran la necesidad de conocer las creencias de los maestros de manera que se puedan promover cambios en las mismas que faciliten el éxito de las reformas educativas.

Similarmente, la búsqueda de alternativas para favorecer el aprendizaje de los alumnos ha llevado al desarrollo de investigaciones específicas sobre los procesos de cambio en los docentes (Fullan \& Miles, 1992; Fullan \& Stiegelbauer, 1991; Richardson, 1990). Las conclusiones generales de las investigaciones indican que para que el cambio sea efectivo y repercuta en las prácticas de enseñanza tienen que: (1) partir de cómo el maestro concibe su propia práctica, (2) llevar al maestro a un análisis crítico acerca de su práctica, (3) que sea participe en la toma de decisiones y en la implantación del cambio y 4) responder al contexto y necesidades particulares del maestro y de los alumnos. 
Macotela Flores, Silvia; Flores Macías, Rosa del Cármen; Seda Santana, lleana: Las Creencias de ...

Es en este punto en particular que planteamos la importancia de estudiar las creencias de los docentes de aula y de apoyo acerca de aspectos que les son comunes. Ante las demandas de una nueva práctica educativa en el contexto de la integración educativa, iniciamos por explicitar en el marco de la presente investigación las creencias de ambos grupos de maestros respecto a tres puntos: (1) lo que significa la escuela como institución, (2) el papel de la escuela en la sociedad, (3) su propia función como docente. En principio, estos tres aspectos son definitorios de las propuestas para la modernización educativa y son la base para entender las creencias de los maestros acerca de la enseñanza, el aprendizaje y la evaluación de los contenidos curriculares.

Sobre estas bases, en el presente trabajo se exploran las creencias de los docentes partícipes en el proceso encaminado a la integración educativa a fin de contar con mayores elementos para contribuir al desarrollo de este proceso. Al igual que en las investigaciones citadas anteriormente, en este trabajo asumimos que las creencias se refieren a proposiciones que son verdaderas para el individuo que las sostiene y que repercuten en sus acciones.

\section{MÉTODO}

Objetivo: Analizar las creencias de docentes de aula y docentes de apoyo en relación a lo que la escuela debe enseñar, así como el papel de la escuela y el maestro para la sociedad.

Tipo de estudio: Descriptivo de campo

Sujetos: Una muestra no-probabilística intencional de 172 docentes de los cuales 100 fueron maestros de apoyo y 72 maestros de aula

Situación: Aulas de las escuelas en donde laboran los docentes en las cuales se realizó la aplicación del instrumento que se describe a continuación.

Instrumento: Tres cuestionarios de once preguntas abiertas. Las primeras tres preguntas son comunes a los tres cuestionarios. Las ocho restantes giraron en torno al aprendizaje y enseñanza de una de tres áreas, lectura, escritura ó matemáticas y se reportan en otros trabajos. Para fines del presente reporte se analizan las tres primeras preguntas: (1) ¿Qué debe enseñar la escuela al individuo? (2) ¿Porqué es importante la escuela para la sociedad?, (3) ¿Porqué es importante el maestro para la sociedad?

\section{PROCEDIMIENTOS:}


Macotela Flores, Silvia; Flores Macías, Rosa del Cármen; Seda Santana, lleana: Las Creencias de ...

Se diseñó una primera versión del instrumento en reuniones del equipo de investigación a partir de la discusión colegiada de las posibles preguntas incluyendo contenido y secuencia. Para esta primera versión se elaboraron 15 preguntas abiertas.

Se solicitó a un grupo de expertos (30 maestros con experiencia en educación básica) que analizaran el instrumento y emitieran juicio al respecto. Para tal efecto se les proporcionó un formato que enunciaba el objetivo del cuestionario, la colaboración que se les estaba solicitando y una sección con las preguntas y dos columnas en las cuales los maestros podían señalar si éstas resultaban claras y apropiadas para el objetivo de la investigación.

El análisis del juicio de expertos condujo a reducir el número de preguntas a 11, así como a revisar la forma en que estaban redactadas las que permanecieron. Se mantuvo el carácter de pregunta abierta. Esta segunda versión se probó en una muestra de 20 maestros de aula y de apoyo. Las respuestas se analizaron en cuanto a la pertinencia para el estudio y se realizaron los ajustes para derivar el instrumento definitivo.

Levantamiento de datos: Con el apoyo de la Dirección de Educación Especial de la Secretaría de Educación Pública (SEP), el instrumento se envió a 6 coordinaciones de educación especial de la ciudad de México, quienes a su vez lo turnaron a distintas escuelas. Cada escuela determinó quienes responderían al cuestionario. Se enviaron 200 cuestionarios, de los cuales se recuperaron los de 72 maestros de aula y los de 100 maestros de apoyo.

Análisis de datos: Para analizar las respuestas a cada pregunta, se empleó una estrategia inductiva. Para cada pregunta un grupo de tres calificadores enumeraró todas las respuestas ofrecidas por los docentes de aula y agruparon las respuestas comunes en categorías. Un segundo grupo de calificadores realizó la misma tarea con las respuestas de los docentes de apoyo. Posteriormente los dos grupos se reunieron y contrastaron la denominación de sus categorías, la definición de éstas y los ejemplos de respuestas. Sobre esta base los calificadores acordaron las categorías para describir los resultados del presente estudio.

La captura y procesamiento de datos se realizó con el programa estadístico SPSS. Se obtuvieron las frecuencias absolutas y relativas de respuesta por categoría y por sub-muestra (docentes de apoyo y docentes de aula), así como las diferencias entre las respuestas de maestros de aula y maestros de apoyo respecto de las categorías.

\section{Resultados}


Macotela Flores, Silvia; Flores Macías, Rosa del Cármen; Seda Santana, lleana: Las Creencias de ...

La mayoría de los maestros ofrecieron para cada pregunta dos o más respuestas, por lo que al realizar la captura éstas podían ubicarse en más de una categoría. En consecuencia, la base de datos se construyó de manera que para cada maestro se pudiera determinar si había respondido o no en alguna de las categorías. A partir de esta estrategia se obtuvieron las proporciones para cada categoría, considerando por separado a la muestra de maestros de aula ( $\mathrm{n}$ $=72$ ) y a la muestra de maestros de apoyo $(n=100)$.

Las Figuras 1 a 3 muestran las categorías derivadas del análisis de contenidos temáticos y la proporción de maestros de aula y de maestros de apoyo cuyas respuestas se ubicaron en dichas categorías. En todos los casos se incluye la categoría de "Otras Respuestas" en la cual se concentraron todas las expresiones que no guardaban relación con la pregunta correspondiente. En las tres figuras los datos se presentan en orden descendente, de las respuestas más frecuentes a las menos frecuentes. En el anexo se encuentran las definiciones para cada una de las categorías de respuesta así como ejemplos de las respuestas ofrecidas por los docentes.

La Figura 1 muestra las seis categorías resultantes para la pregunta. ¿Qué debe enseñar la escuela al individuo? (1. Una formación que le beneficie; 2. Conocimientos; 3. Actitudes y Valores; 4. Beneficio Social; 5. Comportamiento autónomos y reflexivos; y 6. Otras respuestas).

La figura permite apreciar un patrón de respuestas semejante entre los grupos en las cuatro primeras categorías. En ambos grupos las respuestas más frecuentes se ubicaron en la primera categoría. Se aprecian sin embargo, algunas diferencias. Por ejemplo, en el caso de la primera categoría, los maestros de aula respondieron en mayor proporción que los maestros de apoyo. La prueba $t$ para grupos independientes indicó que la diferencia resultó significativa $(\mathrm{F}=7.047 ; \mathrm{p}=.009)$. En la cuarta categoría, la diferencia entre grupos también resultó estadísticamente significativa $(F=16.07 ; p=.000)$ pero a favor de los maestros de apoyo. Aun cuando en las categorías 2, 3, 5 y 6 se aprecia como tendencia, una mayor proporción de respuestas de los maestros de apoyo en contraste con los de aula, en ninguno de estos casos las diferencias resultaron significativas. 
¿QUÉ DEBE ENSEÑAR LA ESCUELA AL INDIVIDUO?

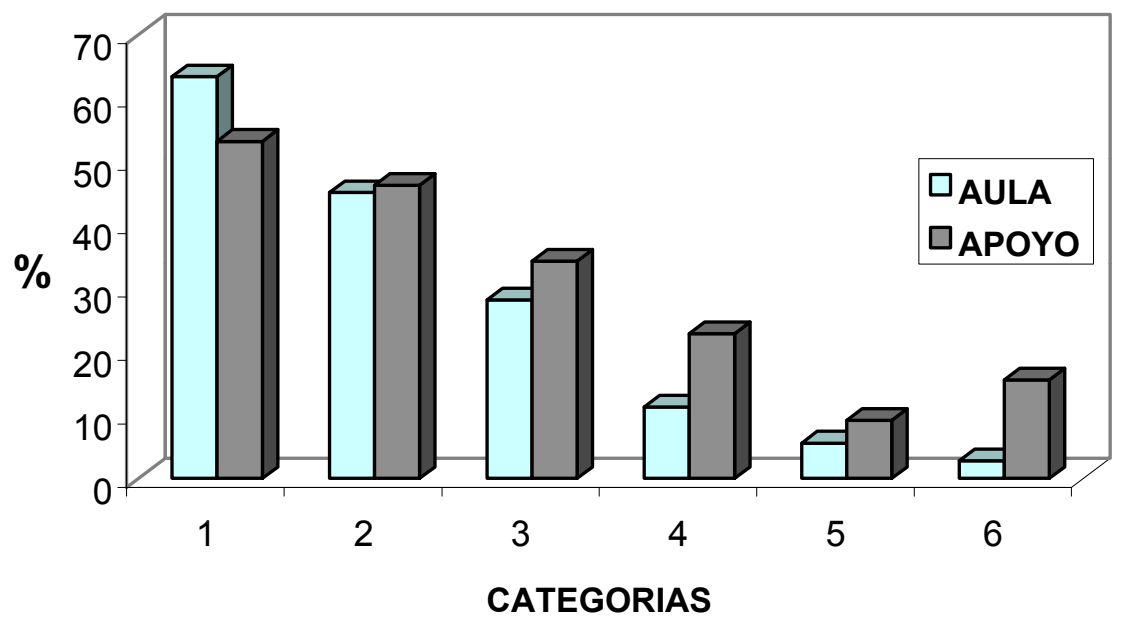

2. CONOCIMIENTOS.

3. ACTITUDES Y VALORES.

4. COMPORTAMIENTOS AUTÓNOMOS Y REFLEXIVOS $(*)$

5. A SERVIR A LA SOCIEDAD

6. OTRAS RESPUESTAS

\section{(*) Diferencia estadísticamente significativa entre grupos}

En la Figura 2 se presentan los resultados correspondientes a la pregunta ¿Por qué es importante la escuela para la sociedad? Para esta pregunta se obtuvieron siete categorías ( 1. Ofrece formación para la vida adulta; 2. Porque preserva la cultura; 3 . Contribuye al desarrollo de la sociedad; 4. Ofrece una formación académica; 5 . Otras respuestas; 6 . Es la instancia educadora legítima; 7. Medio de control del estado).

Nuevamente se aprecia en esta figura la semejanza en el patrón de respuestas de los dos grupos de maestros. No obstante, existen cuatro categorías en donde las diferencias entre grupos 
Macotela Flores, Silvia; Flores Macías, Rosa del Cármen; Seda Santana, lleana: Las Creencias de ...

resultan estadísticamente significativas. En las categorías 1 y 3 los maestros de apoyo responden en mayor proporción que los maestros de aula ( $F=8.095 ; p=.005$ y $F=5.105 ; p=.025$ respectivamente ). En contaste, para las categorías 2 y 7 es mayor la proporción de maestros de aula que responden que la de los maestros de apoyo. ( $F=4.203 ; p=.042$ y $F=12.164 ; p=.001)$. En las categorías 5 y 6 se puede observar una tendencia a favor de los maestros de aula, aún cuando las diferencias no resultan significativas.

¿POR QUÉ ES IMPORTANTE LA ESCUELA PARA LA SOCIEDAD?

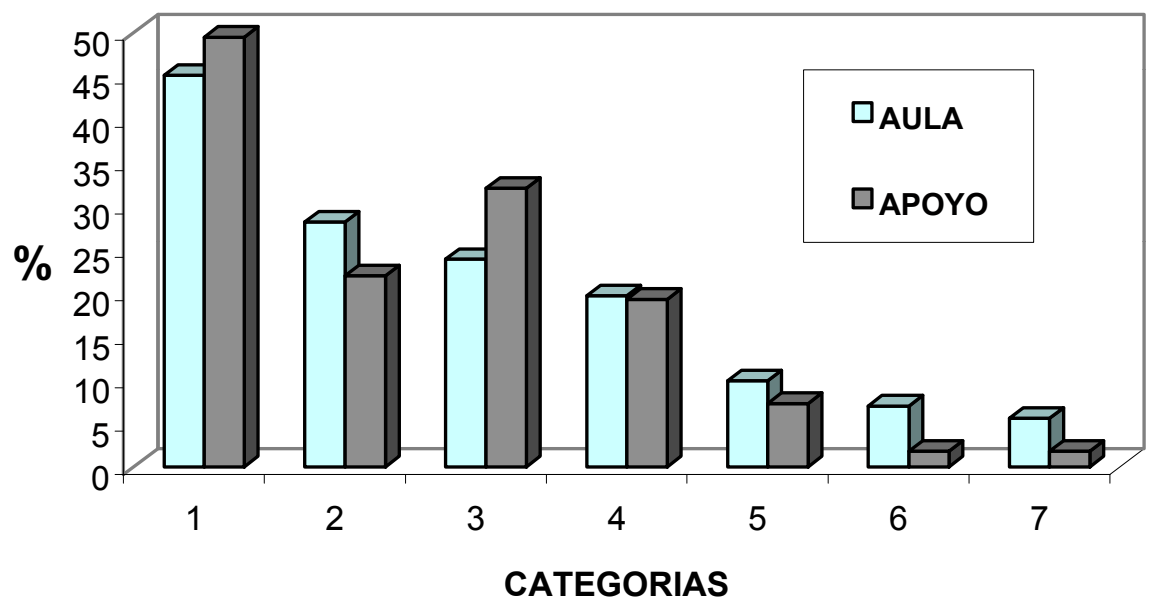


Macotela Flores, Silvia; Flores Macías, Rosa del Cármen; Seda Santana, lleana: Las Creencias de ...

\author{
1. OFRECE FORMACIÓN PARA LA VIDA ADULTA $\left(^{*}\right)$ \\ 2. PRESERVA LA CULTURA $(*)$ \\ 3. CONTRIBUYE AL DESARROLLO DE LA SOCIEDAD $\left(^{*}\right)$ \\ 4. OFRECE UNA FORMACIÓN ACADEMICA: \\ 5. OTRAS RESPUESTAS \\ 6. ES LA INSTANCIA EDUCADORA LEGÍTIMA \\ 7. MEDIO DE CONTROL DEL ESTADO $(*)$
}

\title{
(*) Diferencia estadísticamente significativa entre grupos
}

La Figura 3 ilustra los resultados del análisis de respuestas para la pregunta ¿Por qué es importante el maestro para la sociedad? Las categorías resultantes fueron seis (1. Porque tiene la autoridad para enseñar; 2. Es formador de individuos; 3. Es un ejemplo para los alumnos; 4. Es un agente de cambio social; 5 . Colaborador con la familia y; 6) Otras respuestas.

Una vez más, se observa en la figura la semejanza en el patrón de respuestas entre los maestros de ambos grupos. En las respuestas a esta pregunta, solamente se encontraron diferencias significativas en la segunda categoría ( $F=6.603 ; p=.011)$ en la cual los maestros de apoyo responden en mayor proporción que los maestros de aula. Aun cuando en la primera categoría la diferencia entre los grupos no resulta estadísticamente significativa, se aprecia una tendencia mayor de respuestas de los maestros de apoyo. 


\section{¿POR QUÉ ES IMPORTANTE EL MAESTRO PARA LA SOCIEDAD?}

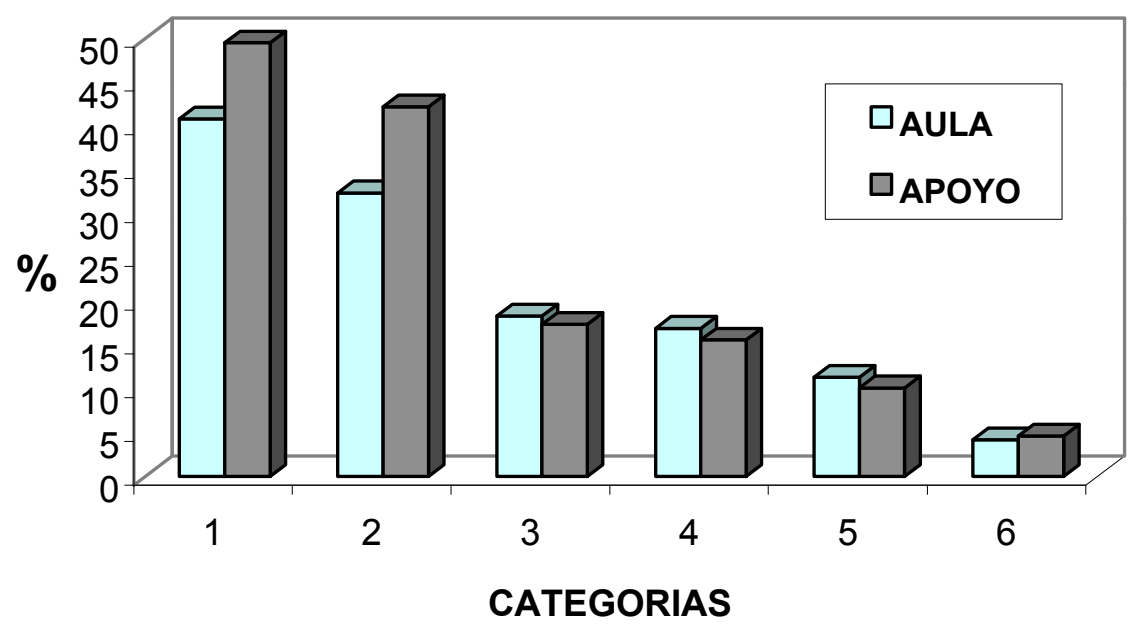

1. TIENE LA AUTORIDAD PARA ENSEÑAR

2. ES FORMADOR DE INDIVIDUOS (*)

3. ES UN EJEMPLO PARA LOS ALUMNOS

4. ES UN AGENTE DE CAMBIO SOCIAL

5. COLABORA CON LA FAMILIA

6. OTRAS RESPUESTAS

(*) Diferencia estadísticamente significativa entre grupos 
Macotela Flores, Silvia; Flores Macías, Rosa del Cármen; Seda Santana, lleana: Las Creencias de ...

\section{DISCUSION}

Con relación a la primera pregunta ¿Qué debe enseñar la escuela al individuo? las respuestas más frecuentes de los docentes de aula y de apoyo (63\% y 53\%) se concentraron en la primera categoría, con expresiones que señalan a la escuela como la institución que proporciona una formación que beneficia al estudiante. El beneficio al que se hace referencia consiste en que el individuo pueda manejar diversas situaciones personales a corto y a largo plazo (problemática cotidiana - afrontar la vida). Esta creencia en ambos grupos, supedita a un papel secundario el compromiso de la escuela por enseñar aquello que contribuya al desarrollo de la sociedad, según se refleja en las proporciones de respuesta de los docentes en la quinta categoría (5\% y $9 \%)$.

Sin embargo, el Programa Nacional de Desarrollo Educativo 1995-2000 (SEP, 1996, p.42) señala "La Ley General de Educación, contempla un mayor acercamiento de las autoridades educativas y la comunidad..." "conviene impulsar las prácticas que nos lleven a una nueva cultura de participación, respeto y co-responsabilidad en torno a la vida escolar". De igual forma se plantea que las reformas educativas tienen como propósito asegurar una educación "que en general, eleve los niveles de calidad de vida de los educandos y de la sociedad en su conjunto". Las respuestas de los docentes no reflejan como creencia importante el vínculo entre escuela y sociedad, ni en esencia el valor social de la escuela.

En congruencia con lo anterior, la segunda respuesta más frecuente para ambos grupos (45\% y $46 \%$ ) corresponde a la enseñanza de conocimientos, superando al número de respuestas de que la escuela debe enseñar a los individuos actitudes y valores (28\% y $34 \%$ ).

Ciertamente, ya que las políticas educativas se han ido encaminando al reconocimiento de esta responsabilidad por parte de las escuelas, se esperaría una mayor proporción de respuestas en esta categoría por parte de los docentes de aula. El Programa Nacional de Desarrollo Educativo 1995-2000 (SEP, 1996) señala "En la educación básica han de adquirirse valores esenciales, conocimientos fundamentales y competencias intelectuales que permitan aprender permanentemente; en ellas se despiertan la curiosidad por el saber y se forman hábitos de trabajo individuales y de grupo".

Al margen de lo expresado, es importante hacer notar que el tipo de respuestas al interior de ambos grupos docentes indica diferentes interpretaciones sobre el concepto de valores. En algunos casos se mencionan valores personales universales tales como "la honestidad y la 
Macotela Flores, Silvia; Flores Macías, Rosa del Cármen; Seda Santana, lleana: Las Creencias de ...

responsabilidad", y en otros se incluyen valores con un carácter comunitario tales como "la conciencia ciudadana y el respeto a la democracia".

La respuesta de ambos grupos de maestros en tercer lugar se refiere a "comportamientos autónomos y reflexivos" (11.3\% y 22.9\%). El hecho de que los docentes de apoyo manifiesten mayor proporción de respuestas que los de aula en esta categoría, puede estar indicando que en la formación y actualización de los primeros se está dando mayor énfasis a estos conceptos en razón del papel que juegan en el apoyo al niño con necesidades educativas especiales (NEE). Sin embargo, se esperaría que los maestros de aula expresaran esta creencia en sus respuestas tanto como los docentes de apoyo, debido al énfasis que han puesto la políticas educativas vigentes al respecto. El Acuerdo para la Modernización Educativa (SEP-SNTE, 1989) establece una plataforma para mejorar la educación: se formarán individuos más preparados y responsables, capaces de aprender, de adecuarse continuamente a nuevos entornos y de afrontar desafíos."

Las respuestas a la segunda pregunta ¿Por qué es importante la escuela para la sociedad?, en su mayoría, sugieren que en ambos grupos de docentes (45.1\% y 49.5\%) existe la creencia de que la importancia de la escuela radica en ofrecer al estudiante una formación para la vida adulta incluida la movilidad social. Es interesante hacer notar que los maestros de aula señalan como segunda respuesta (28\%)la importancia de la escuela como instancia responsable de la preservación de la cultura, a pesar de que sus respuestas no permiten identificar si se está aludiendo a un rasgo individual (individuo culto) o a la cultura como valor social. Por contraste, los docentes de apoyo señalan como segunda prioridad (32\%) la importancia de la escuela como institución que contribuye al desarrollo de la sociedad.

Ambos grupos coinciden en las respuestas que se ubican en cuarto lugar en cuanto a frecuencia $(19.7 \%$ y 19.3\%), la importancia de la escuela como instancia que proporciona al individuo una formación académica. También resulta interesante, aún cuando los porcentajes son bajos, que los maestros de aula mencionen más que los de apoyo, la importancia de la escuela en términos de legitimidad $(7 \%$ y $1 \%)$ y medio de control del estado (5.6\% y $1.8 \%)$.

Al igual que en la primera pregunta, las respuestas sugieren la creencia de que la escuela constituye un factor para la superación individual. Pero además se agrega una creencia subordinada al mencionar a la escuela como elemento de movilidad social. Para el logro de esta movilidad el énfasis se coloca en la enseñanza de conocimientos (básicamente escolares). Es interesante notar que en las respuestas a esta pregunta no aparece el papel de la escuela en lo relativo a una formación ética y cívica. 
Macotela Flores, Silvia; Flores Macías, Rosa del Cármen; Seda Santana, lleana: Las Creencias de ...

Las respuestas a la tercera pregunta ¿Por qué es importante el maestro para la sociedad? en ambos grupos de docentes aluden al papel del maestro poniendo el acento en su carácter de autoridad, como poseedor y transmisor de conocimientos $(40.8 \%$ y $49.5 \%)$, con la particularidad de que su labor se visualiza fundamentalmente hacia el individuo propiamente $(32.4 \%$ y $42.2 \%)$ y en un segundo plano del maestro hacia la sociedad como agente de cambio $(16.9 \%$ y $15.6 \%)$.

En cuanto al carácter del maestro como autoridad y como transmisor de conocimientos, claramente se aprecia la reproducción de un discurso oficial. "El protagonista de la transformación educativa de México debe ser el maestro. Es el maestro quien transmite los conocimientos, fomenta la curiosidad intelectual y debe ser ejemplo de superación personal" (Programa Nacional para la Modernización de la Educación Básica, (Poder Ejecutivo Federal-SNTE, 1992).

Se aprecia nuevamente la congruencia entre las respuestas a esta pregunta y las ofrecidas a las dos anteriores. Por ejemplo, en la primera pregunta los docentes expresan la importancia de que la escuela enseñe conocimientos. En ésta tercera pregunta la creencia mayoritaria consiste en reconocerle al docente la responsabilidad de cumplir con lo que establece el currículo, lo que puede asociarse también a la enseñanza de conocimientos escolares.

Por otro lado, en esta misma pregunta sobresale el bajo porcentaje de respuestas que se refieren a la relación del maestro con la familia en ambos grupos (11.3\% y 10.1\%), no obstante que en el Plan de Desarrollo Educativo 1995-2000 (SEP, 1996) le otorga un papel medular "conviene impulsar las prácticas que nos lleven a una nueva cultura de participación, respeto y coresponsabilidad en torno a la vida escolar" "...Fomentar la vinculación entre los padres de familia, la escuela y la comunidad” (p. 42), “... la escuela es el espacio donde profesores, directores y alumnos comparten la responsabilidad cotidiana de la enseñanza y el aprendizaje" "En cada plantel, los padres de familia, y otros miembros de la comunidad se unen en el objetivo común de participar en la formación de los alumnos.." (p. 43).

\section{CONCLUSIONES}

Como se señaló en la introducción, el presente trabajo forma parte de un proyecto más amplio. Los datos aquí presentados constituyen una de las dimensiones de la etapa diagnóstica de dicho proyecto.

En esta perspectiva conviene subrayar que el análisis de las creencias de los maestros de aula y de apoyo respecto de las preguntas motivo de este reporte constituye un punto de partida 
Macotela Flores, Silvia; Flores Macías, Rosa del Cármen; Seda Santana, lleana: Las Creencias de ...

para determinar qué tanto estas creencias contribuyen al logro de las iniciativas de integración educativa.

Uno de los elementos centrales de dicha iniciativa se encuentra en la premisa de que tanto los docentes de aula como los de apoyo asumirán que su labor habrá de concentrarse en el apoyo a la consecución de objetivos de la educación básica. Para el docente de apoyo, esta situación representa un importante cambio en razón de su historia como parte de un subsistema educativo (educación especial) relativamente independiente de la educación regular. Para el docente de aula también significa un cambio determinante en el sentido de que implica ahora compartir su espacio y responsabilidades con los docentes de apoyo.

Una parte importante de los esfuerzos institucionales se ha encaminado a sensibilizar a docentes de aula y de apoyo respecto a la necesidad de asumir estos cambios. Una forma de dar cuenta del efecto de dichos esfuerzos consiste en determinar qué tanto ambos grupos de docentes comparten hoy creencias básicas respecto al significado de la escuela como institución, al papel de la escuela en la sociedad y la función del docente mismo. En esencia, estos conceptos debieran concebirse como el eje común alrededor del cual puede articularse la labor del maestro de aula y de apoyo.

Si bien las respuestas analizadas indican un alto nivel de coincidencia entre maestros de aula y de apoyo lo cual favorece los procesos de integración, la tendencia a concebir a la escuela como instancia que educa a los individuos para promover su desarrollo personal con poco compromiso social representa a la vez una limitante para estos procesos. El respeto a la individualidad ciertamente forma parte del concepto de educación para la diversidad, exigencia de las iniciativas de integración, pero cuando esto se convierte en una visión individualista, se torna incompatible con la noción de colaboración y de establecimiento de metas comunes.

Por otro lado, los datos sugieren una tendencia general a conceptuar a la escuela como una instancia proveedora y al alumno como el receptor de lo que la escuela provee. Esta creencia más acentuada en los maestros de aula que en los de apoyo representa un motivo de reflexión dada su implicación para la actividad de enseñanza propiamente dicha. No se trata de que sólo el alumno con NEE desarrolle la capacidad de reflexión y de aprender por sí mismo, sino de que esto se fomente en todos y cada uno de los educandos, como meta común por parte de los docentes.

Dicha creencia también conduce a plantear la necesidad de revisar los esquemas de formación de los maestros de aula y de apoyo. Esta formación ha estado a cargo de diferentes instancias formadoras de maestros, aún cuando ambas dependen de la Secretaría de Educación 
Macotela Flores, Silvia; Flores Macías, Rosa del Cármen; Seda Santana, lleana: Las Creencias de ...

Pública. Es necesario que no solamente se plantee la necesidad de que los docentes de aula y apoyo trabajen en colaboración, también es necesario que los programas de formación docente prevean la preparación para este trabajo colaborativo, mediante la inclusión de contenidos relativos a métodos comunes de instrucción y evaluación relativos al currículo de educación básica, así como a través del fomento a la reflexión sobre lo que esto implica (Vaughn \& Schumm, 1995).

Al respecto, Richardson, (1996) en el análisis que realiza del trabajo de varios autores acerca de la relación entre práctica docente y creencias, señala que las interpretaciones de los docentes respecto a la relación del alumno con la escuela son precursoras de sus acciones docentes y sólo pueden cambiar cuando el docente participa en un proceso personal de exploración, experimentación y reflexión. Si bien este proceso personal sin duda debe formar parte de la realidad de quienes ejercen la docencia, también debe constituir un elemento central en los programas de formación docente.

Por su parte Tatto (1998) sostiene que los propósitos de la formación de maestros pueden moldear tanto las creencias de los docentes acerca de lo que resulta apropiado en la enseñanza, como las concepciones acerca de su rol profesional. Retomando a Buchman (1986), la autora afirma que las concepciones acerca del propio papel como docente, a su vez moldean la práctica educativa.

Janney \& Snell (1995) investigaron cómo las creencias y actitudes de los docentes afectan en forma diferencial la adopción de propuestas de integración educativa y los cambios consecuentes en políticas y organización educativa y, cómo la adopción de estas propuestas a su vez, afecta las creencias del maestro. Estas autoras subrayan el hecho de que la adopción de una nueva propuesta depende en gran medida de que el maestro cuente con experiencias de formación en las que él pueda analizar sus creencias acerca de la integración y pueda experimentar y reflexionar sobre lo que implica tener un alumno con necesidades educativas especiales en el aula.

Cabe mencionar que en términos generales, el análisis de las creencias de docentes de aula y de apoyo realizado en el presente trabajo, indica conocimiento del discurso oficial, lo que constituye un paso importante para la adopción de los planteamientos inherentes. Sin embargo, es claro que existen distancias entre el nivel declarativo y la práctica cotidiana, particularmente cuando están implicados conceptos que contrastan con las historias y experiencias personales e institucionales. 
Macotela Flores, Silvia; Flores Macías, Rosa del Cármen; Seda Santana, lleana: Las Creencias de ...

Reconociendo que la integración educativa constituye un proceso y no un fenómeno estático que transcurre de manera automática de un estadio a otro, consideramos que el estudio de las creencias de los docentes, actores principales de esta tarea, constituye un elemento valioso para conocer y comprender de qué manera contribuyen a dicho proceso. El fin último consiste en utilizar este conocimiento con objeto de diseñar mecanismos que conduzcan a generar creencias compartidas, como sustento de la redefinición de responsabilidades y el establecimiento de metas comunes. Sin esto, las posibilidades reales de la integración educativa estarían en discusión.

\section{Referencias Bibliográficas}

AINSCOW, M., Necesidades Especiales en el Aula: Guía para la Formación del Profesorado. Madrid: Narcea, S.A. de Ediciones, 1995.

BENDER, W.N., VAIL, C.O. \& SCOTT, K. Teachers' attitudes towards increased mainstreaming: Implementing effective instruction for students with learning disabilities. Journal of Learning Disabilities. 28 (2), 1995, 87-94.

BORKO, H., \& PUTNAM, R. Learning to Teach. En P. Berliner \& R. Calfee (Eds) $\underline{\text { Handbook of }}$ Educational Psychology, N.Y.: MacMillan, 673-708, 1996.

BRECK, S. (1995). Implementing professional development schools. Seeking a shared vision. Trabajo presentado en la Convención de la American Educational Research Association, San Francisco, CA, Abril de 1995.

CALDERHEAD, J., (1996) Teachers: Beliefs and Knowledge. En P. Berliner \& R. Calfee (Eds) Handbook of Educational Psychology, N.Y.: MacMillan, 709-725, 1996. 
Macotela Flores, Silvia; Flores Macías, Rosa del Cármen; Seda Santana, lleana: Las Creencias de ...

DE WITT, P., BIRRELL, J., EGAN, M.W., COOK, P. F., OSTLUND, M.F., \& YOUNG, J. R. Professional development schools and teacher educators' beliefs: Challenges and change. Teacher Education Quarterly, 25 (2), 1998, 63-80.

FEAR, K. L. Building a democratic learning community within a Professional Development School. Trabajo presentado en la Convención de la American Educational Research Association, San Francisco, CA, Abril de 1995.

FULLAN, M. \& MILES, M. Getting reform right: What works and what doesn't. Phi Delta Kappan, 73 (10), 1992, 744-752.

FULLAN, M. \& STIEGELBAUER, S. The new meaning of educational change. N.Y.: Teachers College Press, 1991.

SACRISTÁN, G. El curriculum: Una reflexión sobre la práctica. Madrid: Ediciones Morata, 1989.

HOOLINGSWORTH, S. (1989). Prior beliefs and cognitive change in learning to teach. American Education Research Journal, 26 (2), 1989, 160-189.

HOLT-REYNOLDS, D. Personal history-based beliefs as relevant prior knowledge in coursework. American Education Research Journal, 29 (2), 1992, 325-349.

JANNEY, R \& SNELL, M. (1995). Integrating students with moderate and severe disabilities: classroom teacher's beliefs and attitudes about implementing an educational change. Educational Administration Quarterly 31, 86 - 114. 
Macotela Flores, Silvia; Flores Macías, Rosa del Cármen; Seda Santana, lleana: Las Creencias de ...

KNOWLES, J. G. Metaphors as windows on a personal history: A beginning teachers' experience. Teacher Education Quarterly, 21 (1), 1994, 37-63.

KNOWLES, J. G. \& COLE, A. L. Through preservice teachers' eyes: Exploring field experiences through narrative inquiry. New York, NY: McMillan College, 1994.

LORTIE, D. C. Schoolteachers: A sociological study. Chicago, IL: University of Chicago Press, 1975.

MACOTELA, S., SEDA, I., \& FLORES, R. Desarrollo y evaluación de un programa-modelo de colaboración entre maestros de aula y maestros de apoyo y su relación con el logro académico en niños de primaria. Proyecto de investigación, México, D. F.: Facultad de Psicología, UNAM, 1997.

MAXSON, M. \& SINDELAR, R. Images revisited: Examining preservice teachers' ideas about teaching. Teacher Education Quarterly, 25 (2), 1998, 5-26.

PAJERAS, M. F. Teachers' beliefs and educational research: Cleaning up a messy construct. Review of Educational Research, 6 (3), 1992, 307-332.

SECRETARÍA DE EDUCACIÓN PÚBLICA. Programa Nacional de Desarrollo Educativo 19952000. México, D.F.: Autor, 1996.

RICHARDSON, V. Significant and worhtwhile change in teaching practice. Educational Researcher, $19(7), 1990,10-18$ 
Macotela Flores, Silvia; Flores Macías, Rosa del Cármen; Seda Santana, lleana: Las Creencias de ...

SECRETARÍA DE EDUCACIÓN PÚBLICA. Proyecto General para la Educación Especial en México, Cuadernos de Integración Educativa, 1, 1994.

SECRETARIA DE EDUCACIÓN PÚBLICA. Documento base de la Conferencia Nacional. Atención educativa a menores con necesidades educativas especiales: equidad para la diversidad, 1997.

SECRETARÍA DE EDUCACIÓN PÚBLICA Y EL SINDICATO NACIONAL DE TRABAJADORES DE LA EDUCACIÓN. Acuerdo Nacional Para la Modernización Educativa. México, D. F.: Autor, 1989.

SECRETARÍA DE EDUCACIÓN PÚBLICA Y EL SINDICATO NACIONAL DE TRABAJADORES DE LA EDUCACIÓN. Programa para la Modernización de la Educación Básica. México, D. F.: Autor, 1992.

TATTO, M. T. The influence of teacher education on teachers' beliefs. Journal of Teachers Education, 49(1), 1998, 66-77.

VAUGHN, S., \& SCHUMM, J.S., (1995). Responsible inclusion for students with learning disabilities. Journal of Learning Disabilities 28, 5, 1995, 264-270. 
Macotela Flores, Silvia; Flores Macías, Rosa del Cármen; Seda Santana, lleana: Las Creencias de ...

\section{ANEXO: DEFINICION DE LAS CATEGORÍAS Y EJEMPLOS DE RESPUESTAS}

\section{Pregunta 1: ¿Qué debe enseñar la escuela al individuo?}

1. UNA FORMACION QUE LE BENEFICIE: Las respuestas se refieren al papel de la escuela con institución que ofrece al individuo conocimientos y habilidades para desarrollarse en la sociedad.

- Enseñar para enfrentar la vida

- Contenidos para desarrollarse en la sociedad

- Formador del individuo en todos los aspectos

- A resolver su problemática cotidiana

2. CONOCIMIENTOS. Las respuestas manifiestan explícitamente que es responsabilidad de la escuela enseñar conocimientos útiles para la escuela y la vida.

- El currículo básico

- Conocimientos encauzados a la práctica diaria

- Conocimientos generales y técnicos que le ayuden a fundamentar su vida escolar

- Conocimientos de todas las áreas del saber humano

3. ACTITUDES Y VALORES. Las respuestas subrayan el papel de la escuela como promotora de normas y hábitos para la convivencia social y el desarrollo personal

- Contenidos que les sirvan para ser una persona con valores

- Hábitos y actitudes para relacionarse con las personas de su medio

- Normas y valores

4. A SERVIR A LA SOCIEDAD. Las respuestas aluden a la responsabilidad de la escuela de formar individuos que contribuyan al mejoramiento de la sociedad. 
Macotela Flores, Silvia; Flores Macías, Rosa del Cármen; Seda Santana, lleana: Las Creencias de ...

- A convivir con sus semejantes, a adaptarse a su medio y mejorarlo

- La mejor forma de adaptarse e integrase a la sociedad

- Respeto a la sociedad en que se vive

5. COMPORTAMIENTOS AUTÓNOMOS $Y$ REFLEXIVOS. Las respuestas indican el compromiso de la escuela por promover la independencia del estudiante y la conciencia crítica sobre su persona y su entorno.

- A pensar, a ser autónomo

- Desarrollar su capacidad para aprender permanentemente y con independencia

- A ser un agente crítico, reflexivo y autónomo

- Que aprenda a aprender

6. OTRAS RESPUESTAS. (Las respuestas no guardan relación ni directa ni explícita con la pregunta)

\section{Pregunta 2: ¿Por qué es importante la escuela para la sociedad?}

1. OFRECE UNA FORMACIÓN PARA LA VIDA ADULTA: Las respuestas se refieren a que la escuela proporciona elementos para el futuro desenvolvimiento laboral y ciudadano del alumno

- Si es eficiente, el individuo adquirirá conocimientos científicos que le ayuden en su desempeño laboral

- Se encarga de la tarea formativa e informativa que dotará al individuo de las herramientas necesarias para ser ciudadano sano y productivo

2. PRESERVA LA CULTURA: La escuela es el medio a través del cual se mantiene la identidad social y cultural

- En un porcentaje alto puede elevar el nivel cultural de la sociedad

- Favorece la transmisión de la cultura 
Macotela Flores, Silvia; Flores Macías, Rosa del Cármen; Seda Santana, lleana: Las Creencias de ...

- Transmite de manera formal valores culturales

- Fortalece el conocimiento de la humanidad

3. CONTRIBUYE AL DESARROLLO DE LA SOCIEDAD. Las respuestas señalan que la escuela coadyuva en la transformación y mejoramiento social

- Para transformarla de acuerdo a las necesidades socio económicas

- Concreta los objetivos que la sociedad pretende para sus ciudadanos

- Para obtener elementos para transformar y mejorar la sociedad en que vivimos

- Para formar una sociedad sana y culta

4. OFRECE UNA FORMACIÓN ACADEMICA: La escuela es responsable de que los contenidos curriculares se impartan.

- Formadora de habilidades cognoscitivas

- Se dan aspectos de formación e información que servirán al alumno

- Se adquieren conocimientos y herramientas para formar adultos preparados

5. OTRAS RESPUESTAS. (Las respuestas no guardan relación ni directa ni explícita con la pregunta)

6. ES LA INSTANCIA EDUCADORA LEGÍTIMA: Las respuestas señalan a la escuela como la institución responsable y autorizada para impartir la educación.

- Es el lugar para que el individuo se desenvuelva en forma integral

- Sin ella sería difícil educar a las personas

- Tiene el objeto de formar al individuo para servirle a la sociedad

7. MEDIO DE CONTROL DEL ESTADO: Las respuestas aluden al mantenimiento del status quo

- Para transmitir una ideología dominante 
Macotela Flores, Silvia; Flores Macías, Rosa del Cármen; Seda Santana, lleana: Las Creencias de ...

- Para que el gobierno mantenga el control

\section{Pregunta 3: ¿Por qué es importante el maestro para la sociedad?}

1. TIENE LA AUTORIDAD PARA ENSEÑAR: Las respuestas manifiestan que el maestro tiene la investidura del conocimiento y la enseñanza.

- Ante la sociedad es el responsable directo e indirecto de la educación.

- Es el portador del conocimiento legítimo

- Es el único que está investido de autoridad pedagógica

- Es la "autoridad" que posee el conocimiento y lo que intenta es transmitirlo

2. ES FORMADOR DE INDIVIDUOS: Las respuestas aluden al papel del maestro como responsable de conducir a los alumnos a integrarse y adaptarse a la sociedad.

- Es el pilar del conocimiento para la formación de todo individuo

- Es un formador de futuras generaciones

- Es el guía que conducirá al individuo para que logre su consolidación.

- Conduce a la comunidad escolar a integrarse y adaptarse a la sociedad

3. ES UN EJEMPLO PARA LOS ALUMNOS: Las respuestas señalan al maestro como modelo a emular.

- Es la imagen a seguir ya que transmite conocimientos educativos y formativos

- Se establece como un guía a seguir

- Da ejemplo a sus alumnos sobre relaciones humanas y actitudes hacia la sociedad

4. ES UN AGENTE DE CAMBIO SOCIAL: Las respuestas se refieren al maestro como promotor de transformaciones sociales.

- Para la preparación de la sociedad, una sociedad preparada puede subsistir 
Macotela Flores, Silvia; Flores Macías, Rosa del Cármen; Seda Santana, lleana: Las Creencias de ...

- Es un líder social que apoya y orienta a la comunidad

- Es el protagonista de los cambios socio económicos que requiere una sociedad

- Parte importante de cualquier cambio que se pretenda llevar a cabo en la sociedad

5. COLABORA CON LA FAMILIA EN LA FORMACIÓN DE INDIVIDUOS: Las respuestas indican el papel conjunto de la escuela y la familia

- Es el primer vínculo exterior entre familia y sociedad

- A través de la figura del maestro la escuela continua una tarea iniciada en la familia

- Es la persona que auxilia a la familia en la formación integral del individuo

- Después de la figura paterna, representa la autoridad y el conocimiento

6. OTRAS RESPUESTAS: (Las respuestas no guardan relación ni directa ni explícita con la pregunta) 